

\section{REVISTA \\ TEORÍA Y PRÁCTICA \\ DE LA \\ ARQUEOLOGÍA HISTÓRICA LATINOAMERICANA}

ISSN: 2250-866X (impreso) | ISSN: 2591-2801 (en línea)

AÑo VIII, VOLUMEN 8, PRIMAVERA DE 2019

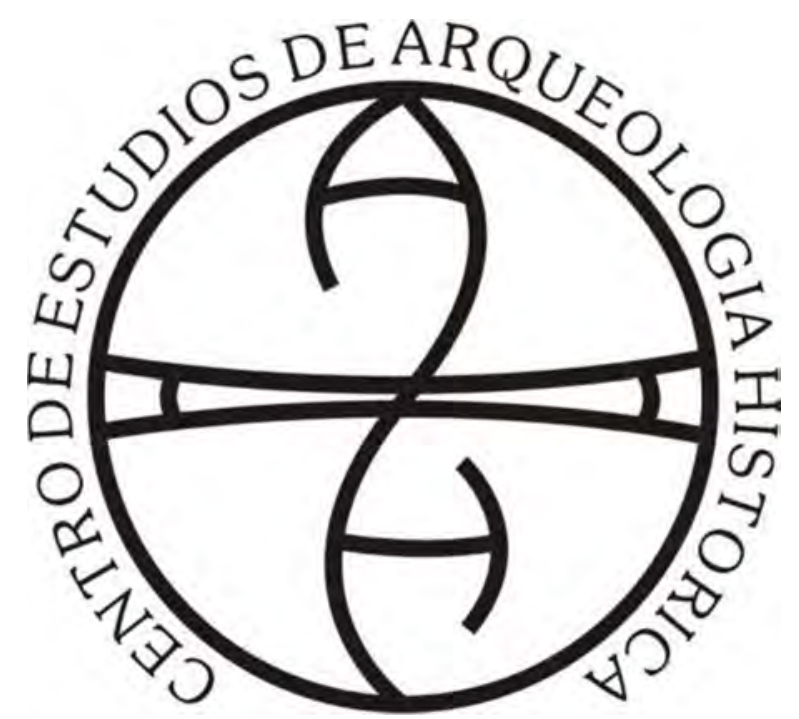

Centro de Estudios de Arqueología Histórica

FACULTAD DE HUMANIDADES Y ARTES | UNIVERSIDAD NACIONAL DE ROSARIO 
(Universidad Nacional de Rosario, Universidad Nacional de Río Cuarto,

Universidad Nacional de San Juan, Universidad de la República, Universidad Nacional de Trujillo)

\author{
AUTORIDADES DE LA UNIVERSIDAD NACIONAL DE ROSARIO \\ RECTOR: Lic. Franco Bartolacci \\ VICE-RECTOR: Od. Darío Macía \\ SECRETARIO GENERAL: Prof. José Goity \\ SECRETARIA ACADÉMICO Y DE APRENDIZAJE: Dr. Marcelo Vedrovnik \\ SECRETARÍA DE CIENCIA TECNOLOGÍA E INNOVACIÓN \\ PARA EL DESARROLLO: Ing. Guillermo Montero.
}

\author{
AUTORIDADES DE LA FACULTAD DE HUMANIDADES Y ARTES \\ DECANO: Prof. Alejandro Vila \\ VICEDECANA: Prof. Marta Varela \\ SECRETARIA ACADÉMICA: Dra. Marcela Coria
}

\author{
AUTORIDADES DEL CENTRO DE ARQUEOLOGÍA HISTÓRICA \\ DIRECTORA: Dra. Ana Rocchietti \\ SECRETARIA: Prof. Nélida de Grandis \\ PROSECRETARIA: Lic. Marianela Bizcaldi
}

DIRECTORAS - EDITORAS:

Dra. Ana Rocchietti y Prof. Nélida De Grandis

SECRETARIA DE EDICIÓN: Dra. Irene Dosztal

Este número es co-edición de las ponencias

del VIII Congreso Nacional de Arqueología Histórica (2018) entre:

Centro de Estudios en Arqueología Histórica: Directora Ana Rocchietti

Centro de Estudios en Arqueología Regional: Director Fernando Oliva

Centro de Estudios en Arqueología Subacuática: Directora Mónica Valentini

Departamento de Arqueología, Escuela de Antropología: Director Fernando Oliva
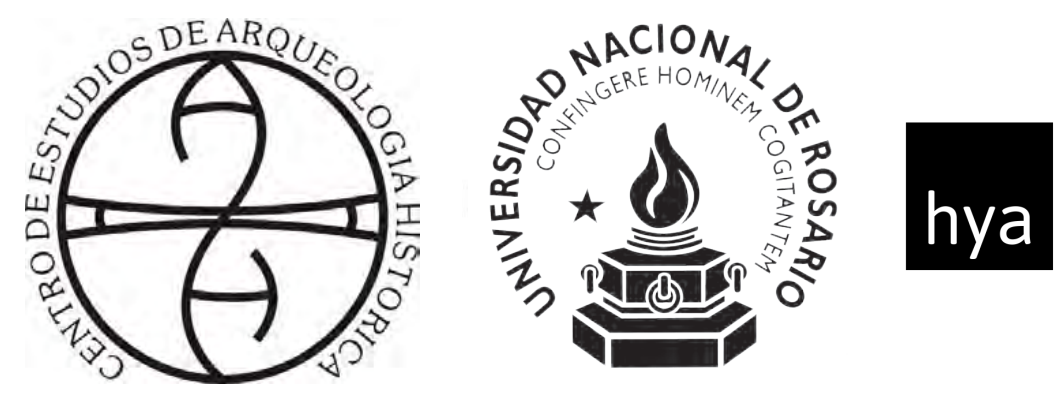

Facultad de Humanidades

y Artes_UNR
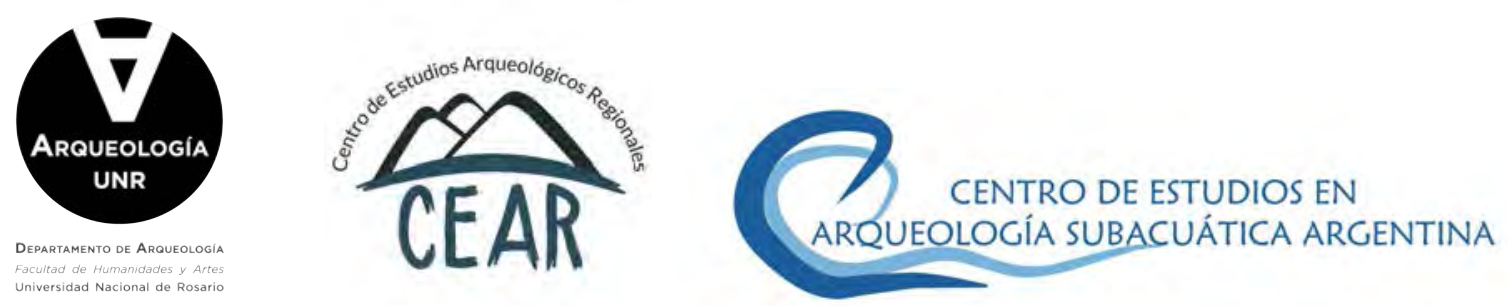
Comité Permanente de los Congresos Nacionales de Arqueología Histórica

Dr. Daniel Schávelzon (Universidad Nacional de

Buenos Aires)

Prof. María Teresa Carrara (Universidad Nacional de Rosario)

Prof. Carlos Baldassarre (Museo Municipal de Río

Grande, Tierra del Fuego) in memoriam

Dr. Mariano Ramos (Universidad Nacional de Luján, CONICET)

Dr. Horacio Chiavazza (Universidad Nacional de Cuyo)

Dra. Ana María Rocchietti (Universidad Nacional de

Rosario, Universidad Nacional de Río Cuarto)

Lic. Facundo Gómez Romero (Universidad Autónoma

de Barcelona)

\section{Comité Científico}

Dra. Tânia Andrade Lima (Universidade Federal do Rio de Janeiro)

Prof. Réginald Auger (CELAT/Département des

Sciences Historiques, Université Laval, Canadá)

Dr. Roberto Bárcena (Universidad Nacional de Cuyo, CONICET)

Dra. Marta Bonaudo (Universidad Nacional de Rosario, CONICET)

Dr. Leonel Cabrera (Universidad de la República, Uruguay)

Dr. Luis María Calvo (Universidad Católica de Santa

$\mathrm{Fe})$

Prof. Juan Castañeda Murga (Universidad Nacional de

Trujillo, Perú)

Dr. Carlos Ceruti (Museo de Ciencias Naturales y

Antropología "Prof. Antonio Serrano". Paraná)

Dr. Horacio Chiavazza (Universidad Nacional de Cuyo)

Dra. Silvia Cornero (Universidad Nacional de Rosario)

Prof. Pedro Paulo Funari (Universidade Estadual de

Campinas, Brasil)

Lic. Jorge A. Gamboa Velásquez (Universidad Nacional

Santiago Antuñez de Mayolo, Perú)

Dr. Eduardo Alejandro García (Universidad Nacional de

San Juan, CONICET)

Prof. Nélida De Grandis (Universidad Nacional de

Rosario)

Dr. Juan Bautista Leoni (Universidad Nacional de

Rosario, CONICET)

Dra. Amancay Martínez (Universidad Nacional de San

Luis)

Dra. Catalina Teresa Michieli (Universidad Nacional de

San Juan, CONICET)

Lic. Fernando Oliva (Universidad Nacional de Rosario)

Ing. Adrián Pifferetti (Universidad Tecnológica
Nacional Regional Rosario)

Dr. Mariano Ramos (Universidad Nacional de Luján, CONICET)

Dra. Ana María Rocchietti (Universidad Nacional de Rosario)

Dr. Daniel Schávelzon, (Universidad Nacional de Buenos

Aires, CONICET)

Dra. Carlota Sempé (Universidad Nacional de La Plata)

Dr. Mario Silveira (Universidad Nacional de Buenos Aires)

Dra. Silvia Simonassi (Universidad Nacional de Rosario)

Dra. Alicia Tapia (Universidad Nacional de Buenos Aires, Universidad Nacional de Luján)

Lic. Mónica P. Valentini (Universidad Nacional de Rosario)

Agrim. Benito Vicioso (Universidad Nacional de Rosario)

\section{Evaluaron este volumen}

Roberto Bárcena, María Teresa Boschin, Leonel Cabrera, Ulises Camino, María Rosa Carbonari, Carlos Ceruti, Horacio Chiavazza, Nicolás Ciarlo, Silvia Cornero, Eduardo Crivelli, Javier García Cano, Martín Gentinetta, María Laura Gili, Carlos Landa, Matilde Lanza, Melina Malandrino, Sebastián Pastor, Victoria Pedrotta, Josefina Piana,

Mercedes Podestá, Mariano Ramos, Daniel Schavelzon, Diana Tamburini, Mónica Therrien, Mónica Valentini y María Teresa Boschin

\section{Diseño y diagramación}

Eugenia Reboiro

(eugenia.reboiro@gmail.com)

\section{Curadoría}

Ana Rocchietti e Irene Dosztal

Foto de tapa: imagen del texto de Croce.

\section{Propietario responsable:}

Facultad de Humanidades y Artes, Universidad Nacional de Rosario Centro de Estudios de Arqueología Histórica

Entre Ríos 758. Rosario, provincia de Santa

Fe (2000). Argentina.

Telf.: +54 (0341) 4802670

E-mail: ceahunr@gmail.com

Decreto Ley 6422/57 de Publicaciones

Periódicas 


\section{Índice}

Editorial.

Arqueología histórica: programa de investigación y dimensiones epistemológicas...9 Ana María Rocchietti

De lozas, iglesias y machaqueños. Primeros pasos hacia una arqueología histórica en la cuenca norte del río desaguadero (La Paz, Bolivia).

Salvador Arano Romero

Identificación arqueológica de acciones militares en el campo de batalla de Cepeda, 1859.

Juan B. Leoni, Lucas H. Martínez, Cecilia Arias Morales, Daniela Cadenas,

Faustino Godoy, Mauro Ganem, María de la Paz Blanche y Héctor Meletta

Para una historia de la arqueología urbana en Buenos Aires (1848-1910) .59

Daniel Schávelzon y Francisco Girelli

Definiendo la cultura material colonial a partir de colecciones arqueológicas del Museo de La Plata.

Melina Bednarz, Julieta Penesis y Ana Igareta

La tangibilidad del conflicto: Arqueología del presente en las fronteras del norte santafesino....

Paula Del Río y Silvia Cornero

Arqueología histórica: evidencia material en el paisaje del establecimiento jesuita "La saladilla” (quebrada de Saladillo, La Rioja, Argentina). Juan Carlos Giuliano, Maira Ayelén Carrizo y María Soledad Gheggi

Graffiti ¿evidencia arqueológica o acto vandálico?. Camila Oliva

Arqueología histórica de los sistemas hidráulicos de la Hacienda

Jalpa de Cánovas, Guanajuato, México, y su relación con el sistema-mundo, Siglos XVIII-XIX

José Alberto Aguirre Anaya y Edgar Quispe Pastrana 
Paseo del bajo: una trinchera en la historia de Buenos Aires.................................143 Federico Martín Croce

Diferentes concepciones del patrimonio y su aplicación a la Casa Histórica de Tucumán también llamada Casa de la Independencia........................................155 Guillermo Etchevers

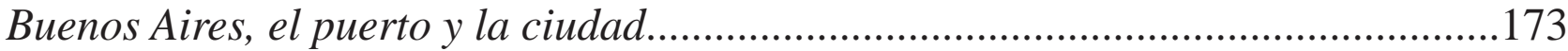
Mónica P. Valentini y Javier García Cano 


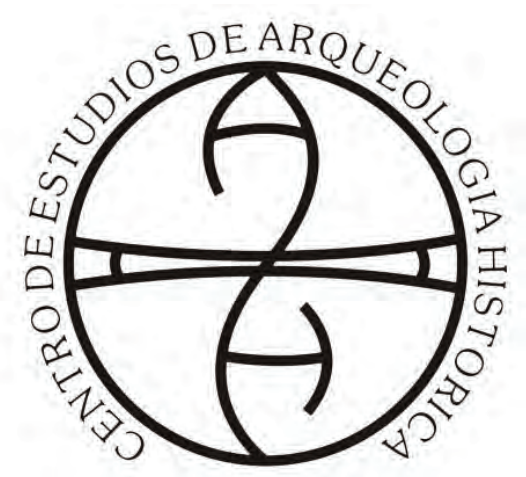

Centro de Estudios de Arqueología Histórica

Universidad Nacional de Rosario
Teoría y Práctica de la Arqueología Histórica

Latinoamericana | Año VIII. Vol. 8 | 2019

Revista del Centro de Estudios de Arqueología

Histórica, Facultad de Humanidades y Artes,

Universidad Nacional de Rosario

https://teoriaypracticaah.unr.edu.ar/index.php/index

ceahunr@gmail.com

ISSN en línea: 2591-2801

ISSN versión impresa: 2250-866X

\title{
PARa UnA historia de la ARQUeología URbana EN BUENOS Aires (1848-1910)
}

\author{
Daniel Schávelzon* y Francisco Girelli**
}

\section{Resumen}

La arqueología urbana se ha diferenciado de la histórica durante los últimos decenios, logrando llegar a constituirse como un campo que estudia la ciudad en todas sus dimensiones y escalas. Para definir su existencia es necesario demostrar que no sólo hay una teoría internacionalmente establecida, sino que a nivel local tiene una historia, un desarrollo, así como ya se lo ha hecho para la arqueología histórica. Un proceso que va desde las primeras experiencias casi inconscientes de su significado hasta su crecimiento actual como territorio académico. Este estudio revisa algunos de los primeros casos que llegaron a los medios de comunicación nacionales desde mediados de siglo XIX hasta principios del XX, con artículos de quienes se hicieron cuestionamientos sobre la cultura material que se encontraba en la tierra en obras de construcción, las publicaron, intentaron respuestas, cruzaron la documentación con los objetos y lograron que el tema tomase carácter público. Cerramos la etapa con el primer caso donde participó un profesional arqueólogo con lo que se inició una fase diferente.

Palabras clave: Arqueología Urbana, Territorio académico, Cultura material urbana

\begin{abstract}
Urban archeology has been differentiated from the historical one during the last decades, managing to become a field that studies the city in all its dimensions and scales. To define its existence it is necessary to demonstrate that there is not only an internationally established theory, but that at the local level it has a history, a development, as it has already been done for historical archeology. A process that goes from the first almost unconscious experiences of its meaning to its current growth as an academic territory. This study reviews some of the first cases that reached the national media from the mid-nineteenth century to the early twentieth century, with articles that were questioned about the material culture that was on earth in construction sites, published, they tried answers, they crossed the documentation with the objects and they got that the subject took public character. We close the stage with the first case where a professional archaeologist participated, which started a different phase.
\end{abstract}

Keywords: Urban Archeology, Academic Territory, Urban Material Culture

* Centro de Arqueología Urbana (CAU-IAA-UBA) - CONICET, dschavelzon@fibertel.com.ar

** Centro de Arqueología Urbana (CAU-IAA-UBA) - CONICET, francisco_girelli@hotmail.com 
Teoría y Práctica de la Arqueología Histórica Latinoamericana | Año VIII. Vol. 8 | 2019

ISSN en línea: 2591-2801 | ISSN versión impresa: 2250-866X

\section{Presentación}

La construcción de un campo disciplinar en la ciencia tiene etapas que son las habituales de todo crecimiento. Una de ellas es el momento en que se comienza a publicar compilaciones bibliográficas y a encontrar que sí existe una producción específica en ese territorio, aunque dispersa. Existe una secuencia que justifica ese nuevo campo que no ha sido definido. Hay otro momento en el que se hace una primera historia de la especialidad. Sólo más tarde vendrá un marco conceptual y metodológico que lo inserte en un espacio de reconocimiento. Y finalmente el establecimiento de instituciones académicas específicas y la aceptación por las más amplias.

Esto ya ha pasado en la arqueología histórica en la Argentina pero no en la urbana, la que aun lucha por ser comprendida en su identidad. Resulta curioso que al revisar la historia del tema encontremos que desde la primer etapa (recopilar lo existente) hasta la segunda (historiar el tema) se hayan tocado pocos ejemplos de Buenos Aires y sí muchos en el resto del país. A la inversa de cómo resultó la historia a partir de la consolidación de las instituciones, en que Buenos Aires es la ciudad que presenta mayor tradición de trabajo continuo (33 años), seguida por Mendoza y su Área Fundacional (28 años) al igual que Rosario (30 años), por citar algunos casos conocidos, mientras que otros aun no lograron consolidarse.

Una revisión de archivos permitió encontrar una importante cantidad de casos olvidados para construir una historia de la arqueología urbana de Buenos Aires. Sucesos entre la segunda mitad del siglo XIX e inicios del XX: hallazgos fortuitos, trucados o intencionales que en su momento no sólo fueron cruzados con los documentos históricos disponibles, sino que también fueron usados para explicar la ciudad, no la historia o lo que decían los textos. Esto se inició en 1848 con los túneles hallados en una casa vecina a la de Juan Manuel de Rosas y se cerró con la intervención de Juan Ambrosetti en la Casa Rosada en 1905; lo que pensábamos era el ejemplo inicial terminó siendo el fin de una etapa. Un circuito que se inició haciendo lo que se podía por quien quisiera hacerlo, hasta el primer llamado a un profesional para que interviniera y excavara el sitio. Entendemos que esta historia no se hace sólo con los eventos sino con los hechos que la comunidad reconoció, que se publicaron y difundieron.

La explicación del período pasa por lo sucedido en la Generación de 1880, la consolidación de un grupo de élite que necesitaba construir su propia historia blanca y occidental para establecer la nueva Nación, la institucionalización con el Museo Nacional de Historia y con el de La Plata, la destrucción de la mal llamada “Gran aldea”, sin registrarla o estudiarla siquiera, la eliminación de los grupos minoritarios desde los pueblos originarios a los afros o el gaucho, y el establecimiento de las formas de apropiación de la naturaleza (zoológico, botánico) y de la cultura (museos, academias).

\section{Los primeros casos en Buenos Aires}

\section{Túneles («mina de explosión») en La Gaceta Mercantil - Supuesto atentado contra Rosas en su casa de Moreno 550 (1848)}

El primer caso en Buenos Aires de lo que hoy definiríamos como un trabajo de arqueología urbana se remonta a 1848. Mientras se realizaba una excavación para la instalación de un pozo ciego en los fondos de una casa ubicada en la calle Belgrano $N^{\circ} 93$ (actual 335) los operarios se sorprendieron por el hallazgo de una estructura subterránea, identificada a priori como una "mina" excavada en la tosca. Debido a la proximidad del hallazgo con los fondos de la casa de Juan Manuel de Rosas, que tenía su frente sobre la actual calle Moreno al 550, se interpretó la estructura como parte de un intento de atentado 
contra el Restaurador. El caso está registrado en dos extensos artículos del diario La Gaceta Mercantil (Anónimo, 17 de abril de 1848; 16 de mayo de 1848). La denuncia del hallazgo derivó en una intensa investigación policial que involucró el interrogatorio a todos los vecinos, propietarios y constructores que trabajaron o habitaron el predio en sus últimos años. Se encargaron también distintos peritajes, entre ellos al conocido arquitecto Felipe Senillosa, a un minero llamado Samuel Weigel, y a otros especialistas, que levantaron planos y relevamientos. Uno de los peritajes contiene una descripción minuciosa de los objetos hallados en el interior de la "boca-mina”, tomando como hallazgo incluso fragmentos mínimos de madera o piedra, con una metodología muy precisa donde se indica la relación estratigráfica de cada objeto (Figura 1).

Finalmente los especialistas concluyeron que dicha estructura tenía al menos veinte años de haber sido excavada, razón por la cual no podía tener vinculación con un intento de atentado a Rosas. Incluso con la investigación encontraron que existían otras estructuras de este tipo en la misma manzana, y otros predios que ya eran conocidas en Buenos Aires, como las que había debajo de la iglesia San Ignacio y otra de la época de las invasiones inglesas (Saguí, 1874). Se descartó la hipótesis del atentado, pero no se pudo determinar cuál era la función del túnel. Este artículo de La Gaceta Mercantil, fue el primero en tratar el tema de los túneles en Buenos Aires, el que es evidente que ya existía en el imaginario popular.

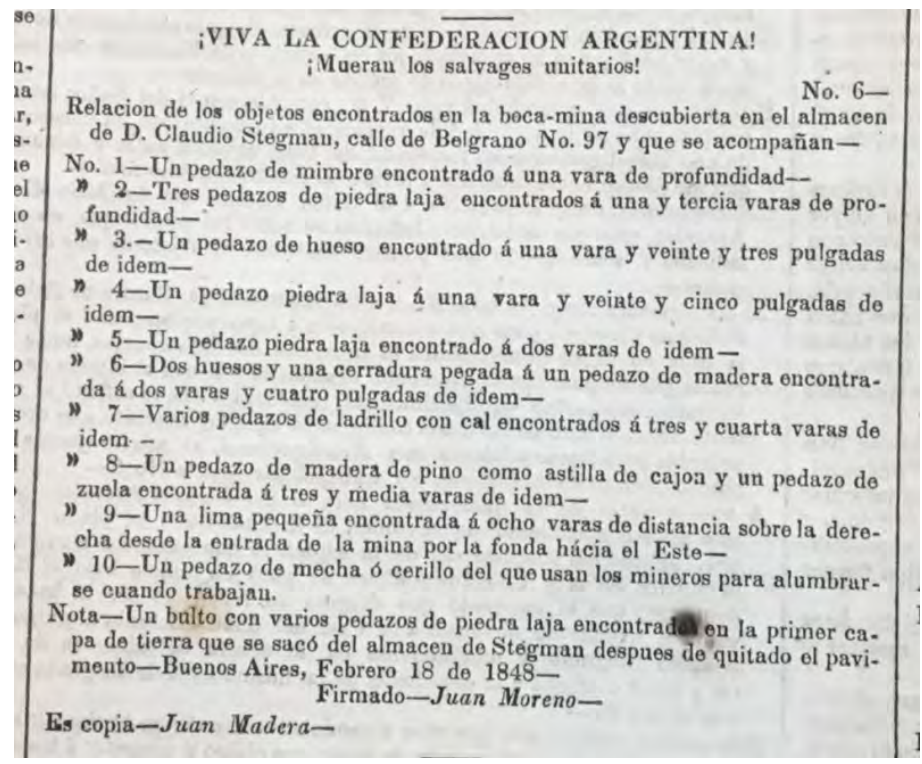

Figura 1. Descripción de los objetos hallados en el interior de la "boca-mina” donde se indica la relación estratigráfica de cada objeto (Anónimo, 16 de mayo de 1848: 2)

\section{Supuesto hallazgo de piedras talladas en la iglesia San Ignacio (1859)}

Durante unas obras de remodelación hechas en el claustro de la iglesia San Ignacio en 1859 supuestamente se encontraron debajo del portal de acceso al claustro, ubicado junto al atrio, un conjunto de piedras talladas: una con la fecha 1675, otra con la cara de un ángel, y la más grande corresponde al torso de San Ignacio de Loyola perteneciente a una talla de gran formato. Dado el hallazgo, el arquitecto de la obra, Miguel Barabino, las colocó sobre el muro de la galería por sugerencia del rector de la iglesia, donde permanecen (Figura 2). La historia está documentada en la época (Quesada, 1865), apenas seis 
años después de la obra, y por una placa de mármol grabada que se colocó junto a las piedras explicando el hallazgo. Tomamos a éste como el primer caso en Buenos Aires donde un hallazgo material contrapone la historia documental oficial (se tomó como la fecha fundacional de la iglesia a la piedra con la fecha), el que luego se incorporó al edificio para su exposición.

En realidad este caso correspondería más a un antecedente ya que suponemos se trata de un mal entendido en la época. Dada la calidad de las tallas y el tipo de piedra es difícil pensar que correspondan a una época primitiva de la misma iglesia. Seguramente se trataba de piedras que estaban en el colegio hacía tiempo, traídas de las misiones u otros lugares de la Compañía de Jesús luego de su expulsión en 1767.
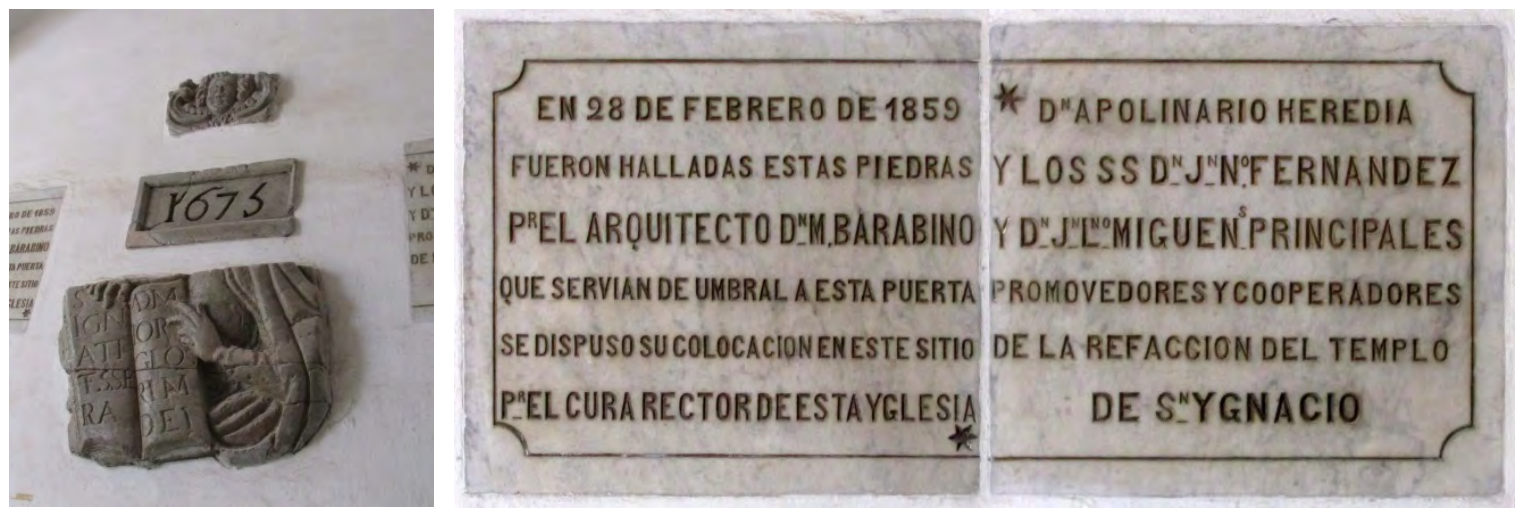

Figura 2. Vista de las piedras y la cartela colocadas sobre el muro lateral del claustro de la iglesia San Ignacio (Fotos del autor, 2017)

\section{Túneles y subterráneos misteriosos (1881 - 1893)}

El caso de los túneles o construcciones subterráneas fue el tema que despertó por primera vez el interés por la arqueología entre los habitantes de la ciudad. Estos se plasmó en cientos de notas y artículos periodísticos que dieron lugar a múltiples interpretaciones.

El tema ya estaba instalado hacia la segunda mitad de siglo XIX: cuando Pierre Benoit realizó las reformas en el Cabildo en 1881 le avisaron antes de iniciar las obras que en las excavaciones que iba a realizar posiblemente iba a encontrar túneles o estructuras subterráneas, de lo cual no encontró nada tal como consta en el informe presentado (Coquet, 1882).

El primer artículo periodístico dedicado específicamente al tema de los túneles es de la revista popular Caras y Caretas de 1904. Se presentaron distintos hallazgos de cisternas y tramos de túneles que ocurrían durante excavaciones por obras nuevas en la ciudad, allí se publicó por primera vez los hallazgos de Burmeister en la Manzana de las Luces, ocurridos una década antes en 1893 (Vidal, 26 de marzo de 1904). A partir de esta publicación el tema de los túneles tuvo gran repercusión en los medios y se instaló en el imaginario porteño como un tema misterioso. Volvió a reaparecer en la prensa con gran cantidad de artículos e interpretaciones en la década de 1920 entre quienes alentaron el misterio y quienes lo refutaron (Schávelzon, 2005). 


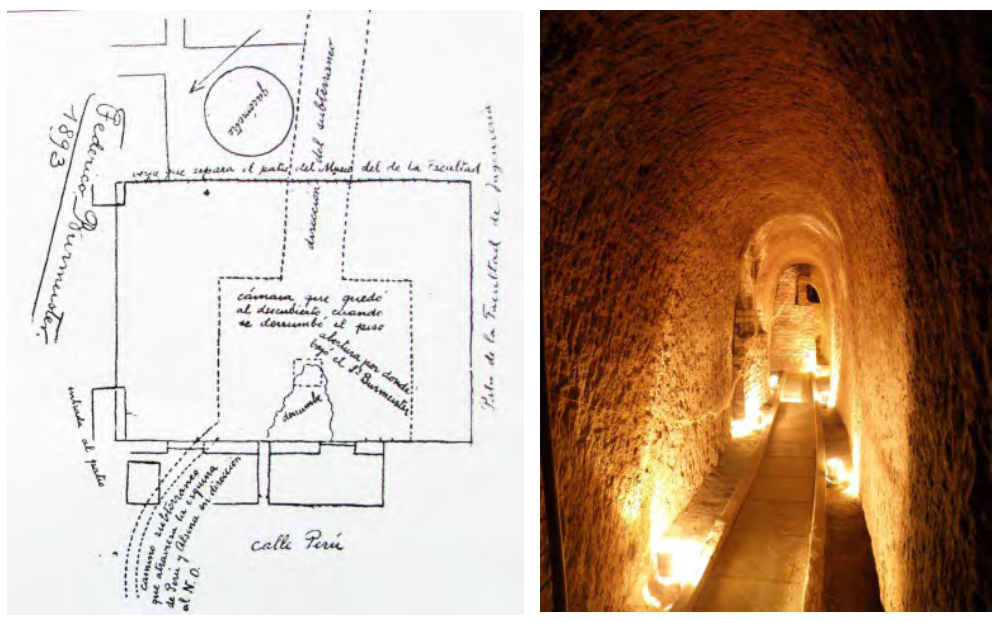

Figura 3. Plano del primer hallazgo de túneles en la Manzana de las Luces relevado por Burmeister en 1893 (Vidal, 26 de marzo de 1904) y foto de un tramo interior de los túneles (Foto: M. Brignani, 2009)

\section{4. "Un arqueólogo del siglo XXX” - Caricatura en Caras y Caretas (1901)}

La caricatura de Cándido Villalobos (Villalobos, 5 de enero de 1901), publicada en Caras y Caretas en 1901, muestra un arqueólogo (afro) del futuro que luego de realizar una excavación arqueológica en Buenos Aires en el año 3000, comunica los hallazgos al público (también afro). Los hallazgos corresponden a objetos de la vida cotidiana de la época y se hace una parodia sobre la interpretación en la práctica de la arqueología: un mate de calabaza es interpretado como una "máquina para zurcir medias", o un tambor de leche como un "instrumento músico usado por los aborígenes en las ceremonias religiosas" (Figura 4).
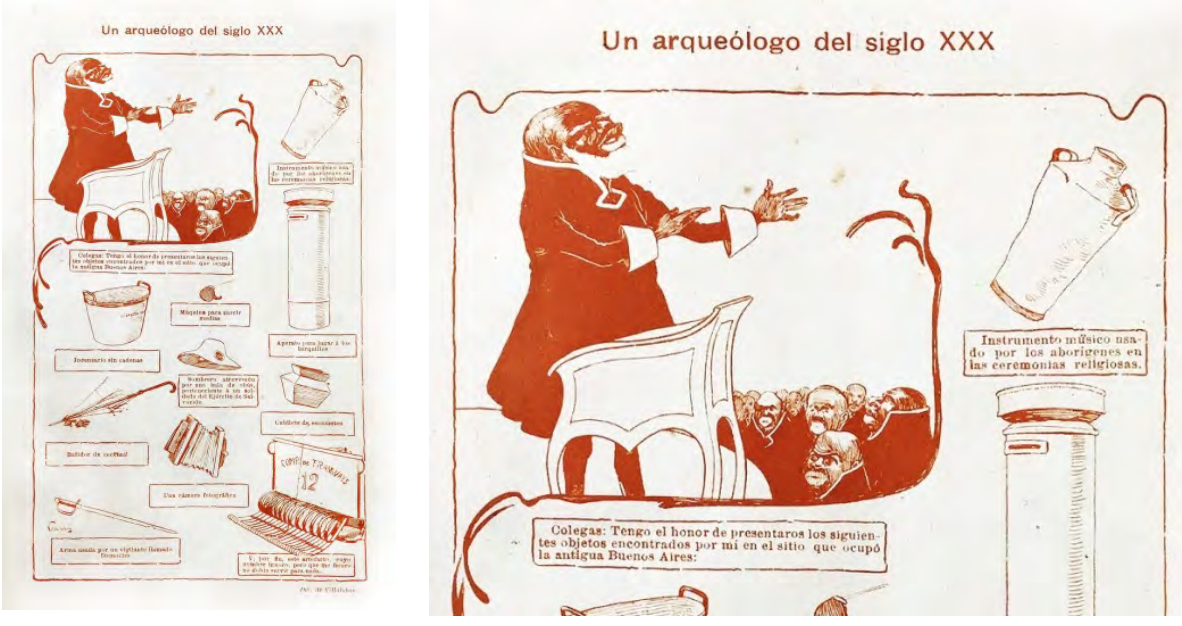

Figura 4. Caricatura de 1901 publicada en Caras y Caretas (Villalobos, 5 de enero de 1901) 


\section{Pasaje 5 de Julio - Invasiones inglesas (1901)}

En 1901, mientras se realizaban trabajos de afirmado y rectificación del pasaje 5 de Julio ubicado en el lateral de la Iglesia Santo Domingo, se produjo el hallazgo de un conjunto de objetos: cuatro hachas, tres balas de gran calibre, un caño de hierro, un precinto de plomo, una llave de puerta y otros objetos y fragmentos no identificados (Figura 5). Los objetos hallados fueron relacionados con los eventos ocurridos durante las invasiones inglesas de 1807 por la cercanía con el conjunto Santo Domingo, epicentro de los enfrentamientos. Una foto de dicho conjunto fue publicada en un artículo de la revista Caras y Caretas (De la Cruz, 17 de agosto de 1901: 29). Se trata del primer caso donde un hallazgo material de época histórica es usado para explicar eventos del pasado reciente.
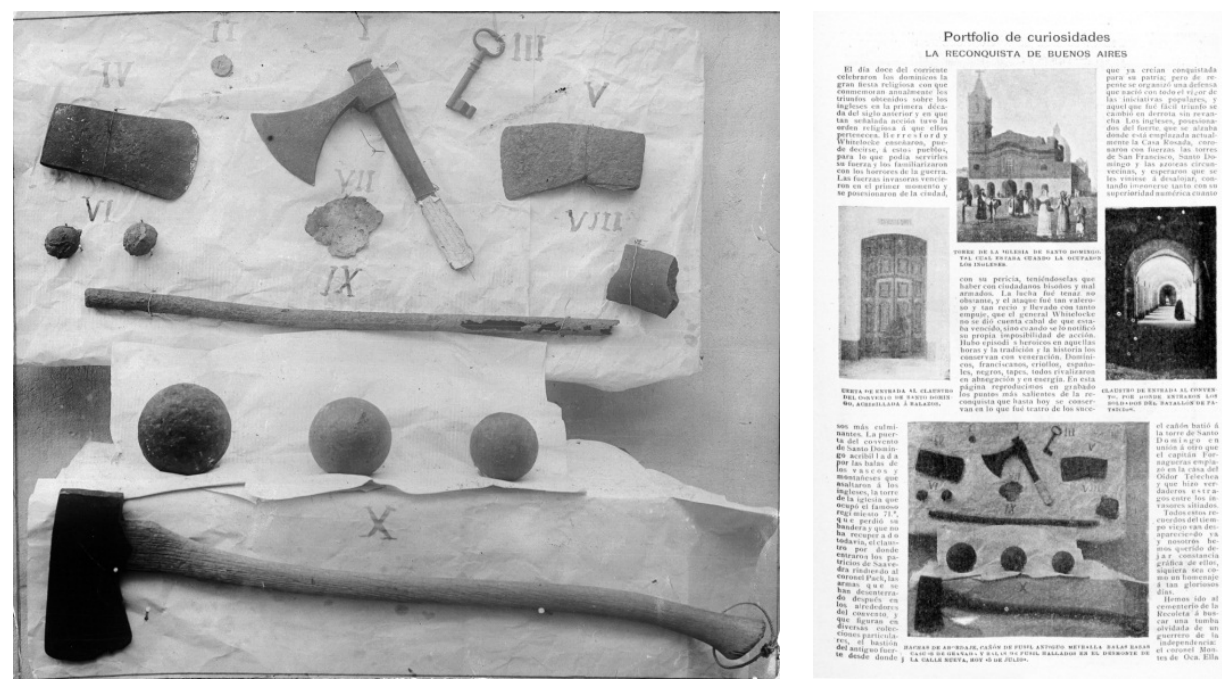

Figura 5. Objetos hallados durante las obras en el Pasaje 5 de Julio (Foto: AGN) y publicación de Caras y Caretas con la interpretación del hallazgo (De la Cruz, 17 de agosto de 1901: 29)

\section{Cañones y "pozo" (cisterna) en antiguo Parque de Artillería (Plaza Lavalle) - Obras de excava- ción y construcción del Palacio de Justicia (1904)}

Durante la demolición del último edificio que quedaba perteneciente al antiguo Parque de Artillería (actual Plaza Lavalle), para la construcción del Palacio de Justicia, se dieron una serie de hallazgos de diversos objetos y “pozos”. Entre ellos se encontró una gran cisterna para almacenamiento de agua, que por alguna razón su función era desconocida en la época, aun cuando se las construía apenas veinte años antes (Figura 6). Otro de los hallazgos fue un cañón de hierro que supuestamente había sido usado en la guerra al Paraguay, y varias municiones (Anónimo, 28 de mayo de 1904). Estos hallazgos se dieron en el año 1904 y a pesar de la repercusión que tuvo en los medios la cisterna fue demolida y el cañón quedó abandonado en el sótano del nuevo Palacio de Justicia. El cañón fue redescubierto por Enrique Udaondo años más tarde, quien lo trasladó al Museo de Lujan (Herz, 1980: 91). 

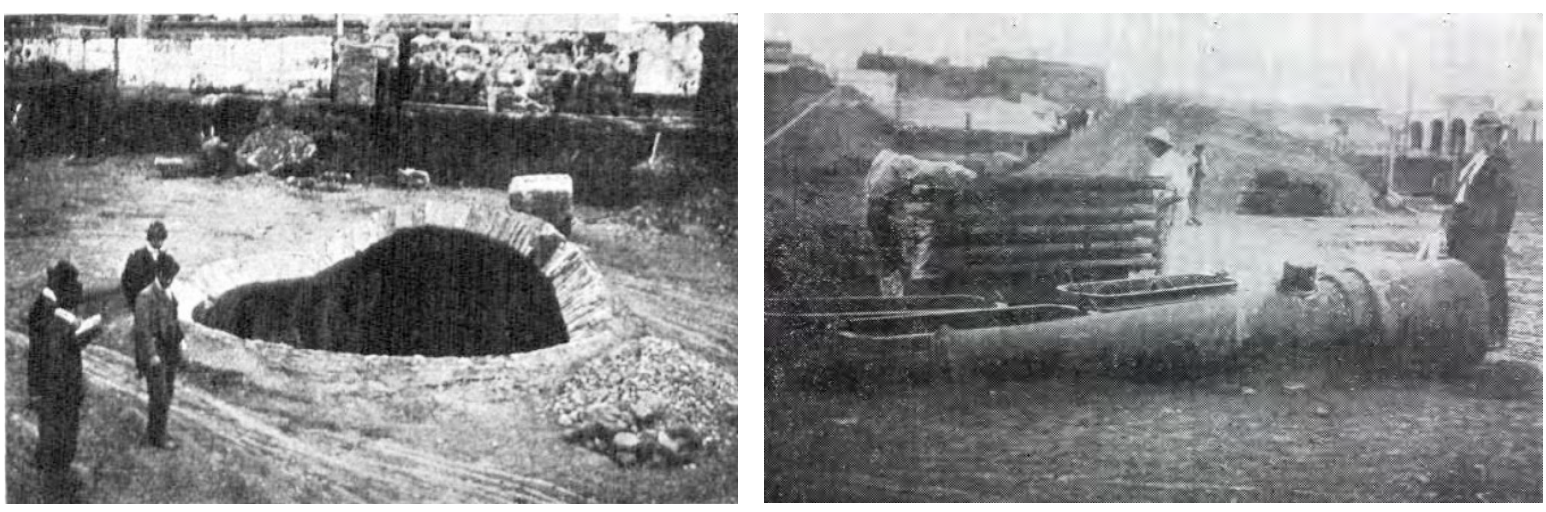

Figura 6. "Pozo” (cisterna) y cañón hallados durante las obras de cimentación del Palacio de Justicia (Anónimo, 28 de mayo de 1904)

\section{Piedras boleadoras en Casa Rosada por Ambrosetti - Rescate durante excavaciones por obras en Patio de las Palmeras (1905)}

El 12 de noviembre de 1905, durante las obras que se efectuaban en el patio principal de la Casa Rosada, conocido como el Patio de las Palmeras, se dio el hallazgo casual de un conjunto de piedras arrojadizas, morteros y huesos (Anónimo, 18 de noviembre de 1905b: 3) (Figura 7). Los responsables de los trabajos dieron aviso a las autoridades quienes convocaron a Juan B. Ambrosetti, quien inmediatamente se dispuso a realizar un trabajo de campo. El hallazgo tuvo gran repercusión en la prensa durante el mes de noviembre (la primera noticia corresponde al diario La Prensa del 15 de noviembre de 1905), y hasta dio para que la revista PBT hiciera chistes acerca de la antigüedad de los funcionarios públicos (Figura 8). Además de algunas fotos, lo único publicado fue la relación observada por Ambrosetti entre esos objetos identificados por él como querandíes con otros del delta del Paraná. Lo interesante de esto es que se trató del primer trabajo controlado por un arqueólogo profesional para estudiar el pasado de Buenos Aires; aunque fue un descubrimiento casual, implicó darle importancia a un hallazgo de este tipo. El conjunto de piedras rescatadas por Ambrosetti en 1905 fue objeto de sucesivas investigaciones por distintos especialistas (Torres, 1911: 75-76; Leguizamón, 1919: 240-243) hasta que en 1925 pasó a formar parte de la colección del Museo Etnográfico. Los materiales fueron redescubiertos por personal del museo en 2012 y nuevamente estudiados (Ammirati et al., 2013).
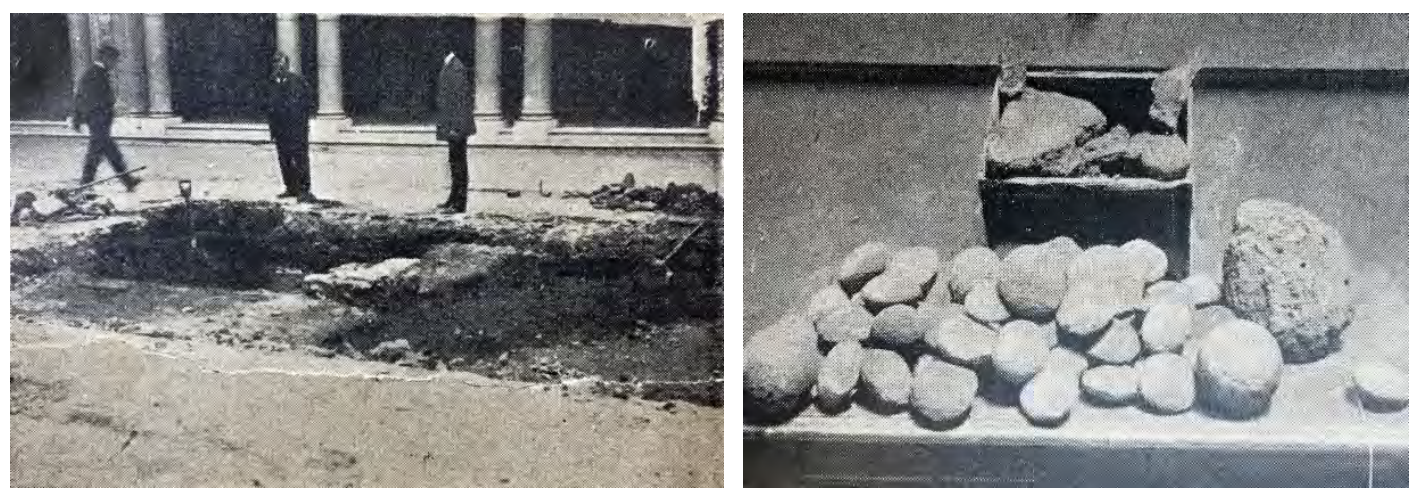

Figura 7. Vista de la excavación que dio lugar a los hallazgos en Casa Rosada en 1905 y las materiales recuperados (Anónimo, 18 de noviembre de 1905a; b: 59) 


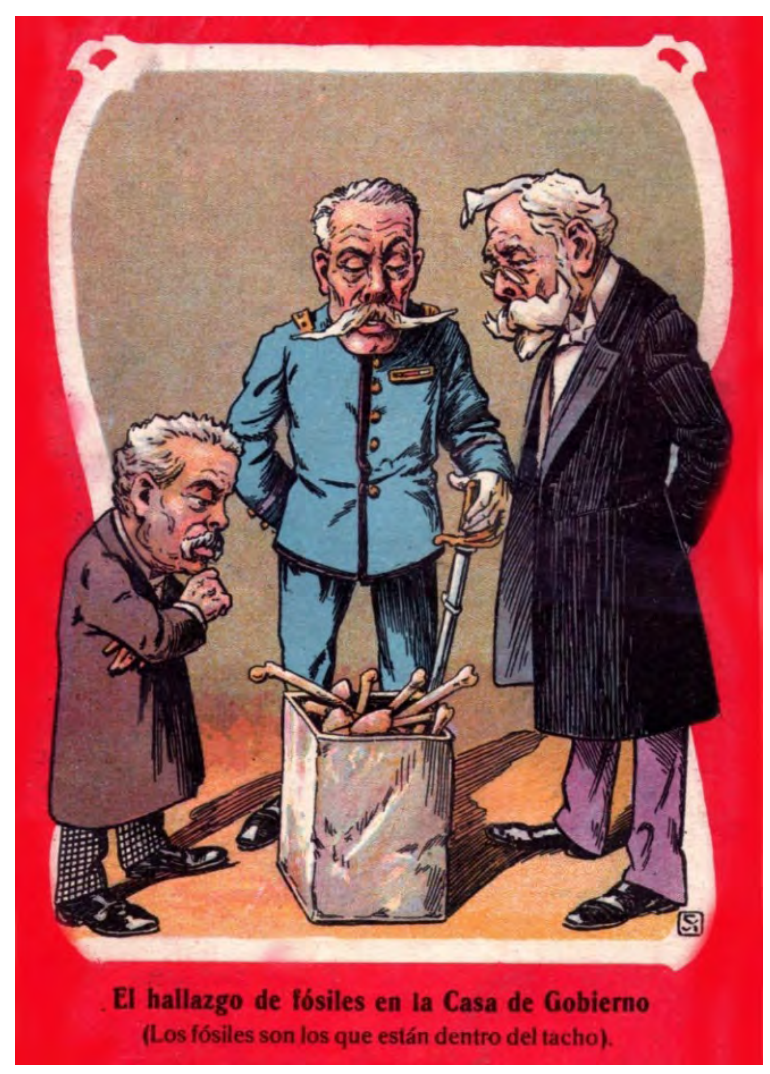

Figura 8. Caricatura política de PBT que refiere a los hallazgos en Casa Rosada (Anónimo, 25 de noviembre de 1905). A la izquierda está representado Juan B. Ambrosetti y a la derecha Manuel Quintana, presidente de la Nación entre 1904 y 1906.

\section{Chiste acerca de hallazgos arqueológicos en Buenos Aires (1907)}

La caricatura de José María Cao (Cao, 31 de agosto de 1907), publicada en Caras y Caretas en 1907 presenta una serie de objetos hallados en "los subterráneos de Buenos Aires”, todos objetos de la vida cotidiana aun en uso en la época, pero donde las interpretaciones sobre su uso y utilidad es fantasiosa (Figura 8). Aunque en este caso se manipula la interpretación como un recurso cómico apelando a la ironía, coincide con la caricatura de 1901 donde se plantea que los objetos cotidianos del presente van a perder su significado a futuro, aun en la era moderna donde existen registros escritos sobre su uso. Así, los restos de un farol de alumbrado público, todavía en uso en la ciudad en esos años y que mostraba la fecha de su fabricación -1890-, se lo interpreta como si fuera un número de serie. 

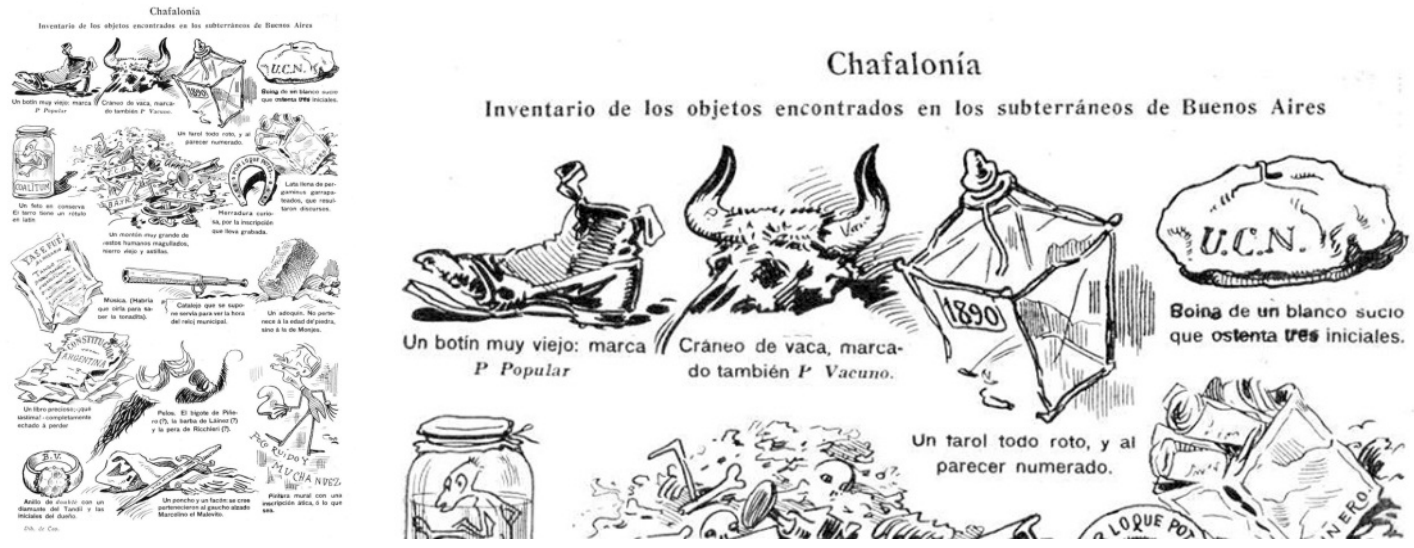

Figura 8. Caricatura de 1907 publicada en Caras y Caretas (Cao, 31 de agosto de 1907)

\section{Cisterna y pozos de la supuesta casa de Rosas hallados durante construcción del edificio Raggio (1909)}

En 1909 se produjo el hallazgo de distintas estructuras subterráneas en la esquina de Bolívar y Moreno, “escondrijos misteriosos” según un artículo aparecido en el diario La Nación (Anónimo, 18 de agosto de 1909). Los hallazgos ocurrieron durante la excavación del subsuelo para la construcción de los Almacenes Raggio, cuya obra empezó a principios de 1909 (Anónimo, 02 de enero de 1909). El interés sobre el caso surgió de asociar el predio con la casa de la familia Rosas-Ezcurra, lo cual estaba equivocado ya que la casa que habitaron ellos era la vecina, sobre Moreno (actual 550). Se describen las construcciones halladas y los objetos encontrados en su interior, entre ellos "un mal pedazo de bayoneta y un plato con el retrato de Napoleón Bonaparte” (Anónimo, 28 de agosto de 1909), mediante los cuales se intentó asignarle cronología al uso de dichas construcciones, que en realidad no eran otra cosa que una cisterna de aljibe, y caños y pozos pertenecientes al sistema sanitario (Figura 9).
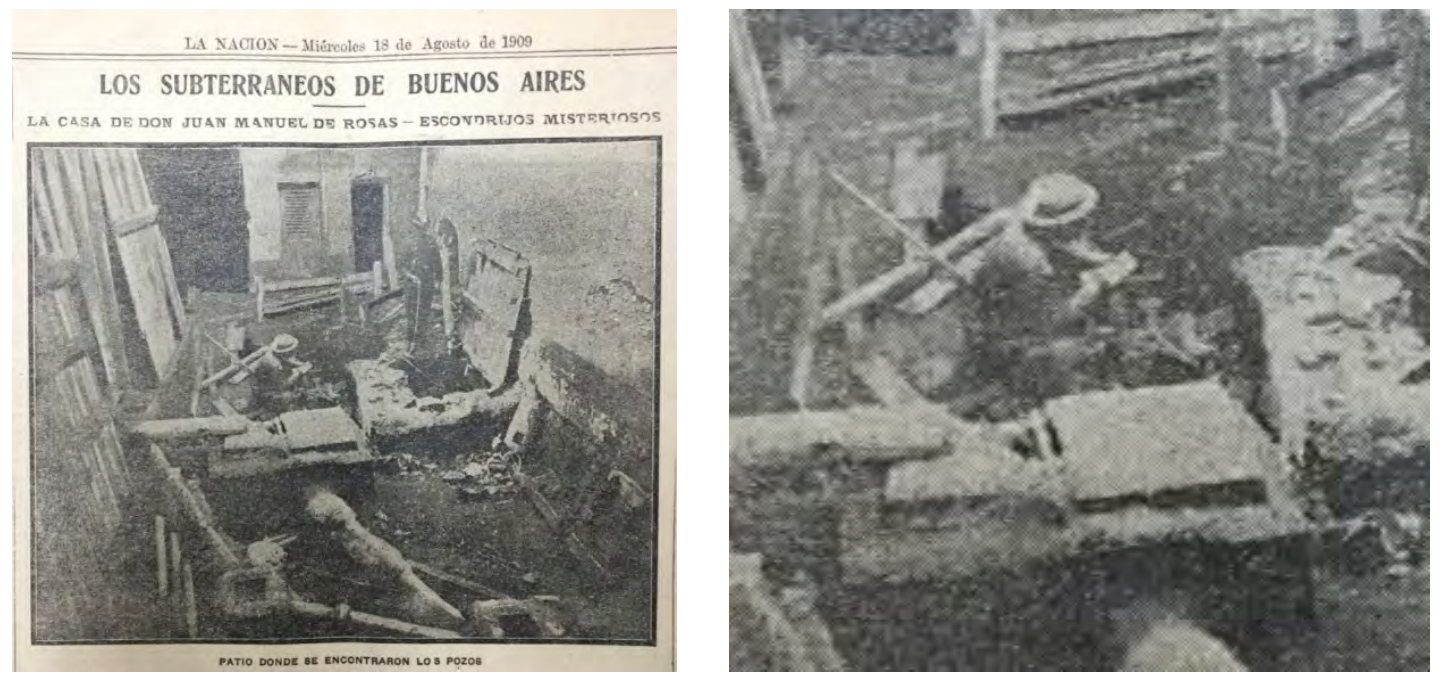

Figura 9. Nota del diario La Nación donde se observa el trabajo de relevamiento sistemático de los “escondrijos misteriosos” pertenecientes a la supuesta casa de Rosas (Anónimo, 18 de agosto de 1909) 
Teoría y Práctica de la Arqueología Histórica Latinoamericana | Año VIII. Vol. 8 | 2019

ISSN en línea: 2591-2801 | ISSN versión impresa: 2250-866X

\section{Un intento de explicación}

Construir la historia de un área del conocimiento es una tarea compleja porque, después de definir su alcance y sustento teórico, implica encontrar los textos que llevaron a su existencia. Y habitualmente, en los inicios, no son más que artículos cortos, anécdotas e incluso chistes, pero muestran que el tema está instalado en la opinión pública. La gente podría mirar con asombro esos hallazgos pero no dejaban de publicarse y el lector entendía por qué existían. La arqueología ya existía para esa época, o al menos estaba creciendo rápidamente, pero sus intereses estaban en lo muy lejano, en la llamada "pre-historia". Estos textos apuntan a un universo cercano, reciente, incluso contemporáneo y se interrogan sobre hechos de la ciudad de los que hay documentación histórica. Pese a eso son recuperados, interpretados y difundidos incluso a contrapelo de lo establecido como el imaginar un futuro de población negra en lugar de blanca. No sabemos cómo lo habrán tomado los “sabios” de su época, los Moreno, Zeballos u Onelli por ciar algunos conocidos. Pero también era parte de la construcción de la historia de un país que se asumía como nuevo, sin pasado lejano. Una historia que no iba más lejos que la inmigración europea o los tiempos coloniales.

La ciudad de Buenos Aires crecía en forma imparable para los estándares de 1900, y muchos necesitaban explicarse a sí mismos quiénes eran, establecer una historia -digna si era posible-, para justificar su existencia, su rol en el mundo. Los historiadores la hicieron en esos mismos años por cierto. Pero a la par de esa historia oficial, de esa explicación, surgían objetos y lugares que contaban historias que no se conocían, o incluso que eran diferentes a lo establecido y eso fue el germen de lo que hoy llamamos arqueología de la ciudad o arqueología urbana. Eran los inicios, el principio de algo que no tenía entidad y no la tuvo por un siglo, pero que ya asomaba con estas curiosas notas y anécdotas.

No podemos dejar de ver que éstas son notas curiosas, casi simpáticas, pero que nos llevan a hacernos preguntas que aun no han sido contestadas. Es notable que una cisterna de aljibe, aunque por cierto muy grande, pero que estuvo en uso una o dos generaciones antes, implica una pérdida de la memoria colectiva casi absurda ya que casi todo el país aun los usaba. O el aceptar fechar una iglesia -nada menos que la más antigua de la ciudad que se conservaba-, por una piedra que llegó de quién sabe dónde, indica la necesidad de más seriedad, por eso lo mismo se ha cuestionado con chistes y humor, se decía mucho sobre eso mismo sin compromiso: cómo se interpretan los restos materiales fuera de contexto y sin documentos históricos o historia oral. El magnífico estudio hecho por simples policías por el túnel de la manzana de la casa de Rosas nos muestra que incluso se estaba usando el método estratigráfico, sin saberlo obviamente, pero las cosas tenían una ubicación en el espacio y el tiempo por su posición horizontal y vertical, incluso el más mínimo fragmento de madera. Un territorio y una historia más que interesante para su investigación.

\section{Referencias bibliográficas}

AMMIRATI, G., ESTÉVEZ, J. M., MARCHEGIANI, M. y REYNOSO, A. (2013). La recuperación de material histórico proveniente del predio del Museo Etnográfico 'Juan B. Ambrosetti' (FFyL, UBA) y su incorporación al acervo arqueológico. Ponencia en $1^{\circ}$ Congreso Latinoamericano y II Congreso Nacional de Museos Universitarios.

ANÓNIMO (17 de abril de 1848). La Gaceta Mercantil, (7335), pp. 1-2.

ANÓNIMO (16 de mayo de 1848). La Gaceta Mercantil, (7358), pp. 1-4. 
Teoría y Práctica de la Arqueología Histórica Latinoamericana | Año VIII. Vol. 8 | 2019

ISSN en línea: 2591-2801 | ISSN versión impresa: 2250-866X

ANÓNIMO (28 de mayo de 1904). Curiosidades. Caras y Caretas, (295), p. 41.

ANÓNIMO (18 de noviembre de 1905a). Un hallazgo arqueológico. Caras y Caretas, (372), p. 39.

ANÓNIMO (18 de noviembre de 1905b). Hallazgo arqueológico. PBT, (61), pp. 3, 49, 55 y 59.

ANÓNIMO (25 de noviembre de 1905). El hallazgo de fósiles en la Casa de Gobierno. PBT, (62), p. tapa.

ANÓNIMO (02 de enero de 1909). El palacio Raggio. Caras y Caretas, (535), p. 153.

ANÓNIMO (18 de agosto de 1909). Los subterráneos de Buenos Aires. La casa de don Juan Manuel de Rosas - Escondrijos misteriosos. La Nación, p. 10.

ANÓNIMO (28 de agosto de 1909). Los subterráneos de Buenos Aires. Caras y Caretas, (569), pp. 55-56.

CAO, J. M. (31 de agosto de 1907). Chafalonía. Inventario de los objetos encontrados en los subterráneos de Buenos Aires. Caras y Caretas, (465), p. 50.

COQUET, J. (1882). Casa de Justicia - Antiguo Cabildo. Memoria presentada por el ministro secretario en el departamento de gobierno á la honorable legislatura de la provincia. Años 1881-1882. Buenos Aires: Imprenta de El Siglo, pp. 197-203.

DE LA CRUZ, J. (17 de agosto de 1901). Portfolio de curiosidades. La reconquista de Buenos Aires. Caras y Caretas, (150), pp. 29-30.

HERZ, E. G. (1980). Historia de la Plaza Lavalle. Colección Cuadernos de Buenos Aires, 53. Buenos Aires: Municipalidad de la Ciudad de Buenos Aires.

LEGUIZAMÓN, M. (1919). Etnografía del Plata. El origen de las boleadoras y el lazo. Revista de la Universidad de Buenos Aires, (XLI), pp. 206-256.

QUESADA, V. (1865). Buenos Aires en 1729. Introducción á las cartas de los jesuitas Gervasoni y Cattaneo. La Revista de Buenos Aires, III (30), pp. 200-213.

VIDAL, B. (26 de marzo de 1904). Una excursión por los subterráneos de Buenos Aires. Caras y Caretas, (286), pp. 30-31.

SAGUÍ, F. (1874). Los últimos cuatro años de la dominación española en el antiguo Virreinato del Rio de la Plata desde 26 de junio de 1806 hasta 20 de mayo de 1810. Memoria histórica familiar. Buenos Aires: Imprenta Americana.

SCHÁVELZON, D. (2005). Túneles de Buenos Aires. Buenos Aires: Editorial Sudamericana.

TORRES, L. M. (1911). Los primitivos habitantes del Delta del Paraná. Buenos Aires: Imprenta Coni.

VILLALOBOS, C. (05 de enero de 1901). Un arqueólogo del siglo XXX. Caras y Caretas, (118), p. 29.

Recibido: 30 de abril de 2019

Aceptado: 14 de mayo de 2019

Para una historia de la arqueología urbana...

SCHÁVELZON y GIRELLI

Páginas 59-69 